\title{
Representações sociais da biossegurança no contexto do HIV/AIDS: contribuições para a saúde do trabalhador
}

\author{
Social representations of biosecurity in the context of HIV / AIDS: contributions to workers' health \\ Representaciones sociales de bioseguridad en el contexto del VIH / SIDA: contribuciones a la salud \\ del trabajador
}

\author{
Ana Paula Munhen de Pontes', Denize Cristina de Oliveira", Virginia Paiva Figueiredo Nogueira'I', \\ Yndira Yta Machado ${ }^{\prime V}$, Renata Lacerda Marques Stefaisk ${ }^{v}$, Marcio Martins da Costa ${ }^{v /}$
}

\begin{abstract}
RESUMO
Objetivo: analisar as representações sociais de biossegurança construídas pelos enfermeiros que atuam com pacientes soropositivos ao HIV. Método: pesquisa qualitativa, exploratória, fundamentada na teoria das representações sociais, realizada em oito instituições de saúde, no município do Rio de Janeiro, através de entrevista com 19 enfermeiras. Os dados foram analisados com o software ALCESTE 4.10. O projeto foi submetido e aprovado por Comitê de Ética em Pesquisa. Resultados: foram construídas duas temáticas: o desenvolvimento da biossegurança em HIV/AIDS na vida pessoal; e a biossegurança em HIV/AIDS e a execução do cuidado de enfermagem. Identificou-se que a autoproteção é um dos elementos fundamentais constitutivos da representação social das enfermeiras sobre biossegurança, assumindo as facetas de autoproteção na vida profissional e na vida pessoal. O uso de luvas de procedimento é caracterizado como a principal medida de precaução no trabalho. Conclusão: destaca-se a influência exercida pela representação social do HIV/AIDS nas ações de autoproteção desenvolvidas pelas enfermeiras.

Descritores: Enfermagem do trabalho; contenção de riscos biológicos; síndrome de imunodeficiência adquirida; enfermagem
\end{abstract}

\section{ABSTRACT}

Objective: to analyze the social representations of biosecurity for nurses who work with HIV-positive patients. Method: qualitative and exploratory research, based on social representations theory, held in eight health institutions, with 19 nurses, in the city of Rio de Janeiro. Data were obtained through interviews and analyzed with the ALCESTE 4.10 software. The project was submitted and approved by Research Ethics Committee. Results: two themes emerged from the analysis: the development of biosafety in HIV/AIDS in personal life and, biosecurity in HIV/AIDS and performing nursing care. It was identified that self-protection is one of the key elements that constitute the nurses' social representation of biosafety, assuming the facets of self-protection in professional and personal life. The use of gloves is featured as the main caution measure at work. Conclusion: stands out the influence of the social representation of HIV/AIDS in the self-protection actions carried out by these nurses.

Descriptors: Occupational health nursing; containment of biohazards; acquired immunodeficiency syndrome; nursing.

\section{RESUMEN}

Objetivo: analizar las representaciones sociales de la bioseguridad construida por enfermeras que trabajan con pacientes VIH-positivo. Método: investigación cualitativa, exploratoria, basada en la teoría de las representaciones sociales, realizada en ocho instituciones de salud, con 19 enfermeras, en el municipio de Río de Janeiro. Los datos fueron recogidos a través de entrevistas y analizados con el software ALCESTE 4.10. El proyecto fue sometido y aprobado en Comité de Ética en Investigación. Resultados: los dos temas surgidos del análisis, fueran: el desarrollo de la bioseguridad en VIH/SIDA en la vida personal y, la bioseguridad en VIH/SIDA y realizar el cuidado de enfermería. Se identificó que autoprotección es uno de los elementos fundamentales que constituyen la representación social de las enfermeras sobre bioseguridad, suponiendo dos facetas: la autoprotección en la vida profesional y vida personal. El uso de guantes es destacado como la principal medida de precaución en el trabajo. Conclusión: se destaca la influencia ejercida por la representación social de las acciones de autoprotección del VIH/SIDA realizado por estas enfermeras.

Descriptores: Enfermería del trabajo; contención de riesgos biológicos; síndrome de inmunodeficiencia adquirida; enfermería.

\section{INTRODUÇÃO}

A equipe de enfermagem caracteriza-se como a categoria profissional que mais sofre acidentes nas instituições de saúde. Este trabalhador encontra-se exposto a diversos riscos oriundos de atividades assistenciais e gerenciais no ambiente laboral. Cumpre destacar que, especialmente, a categoria dos auxiliares de enfermagem apresenta elevado número de acidentes dada sua rotina de trabalho, muitas vezes exaustiva e estressante ${ }^{1-3}$.

'Enfermeira. Doutora. Professora Adjunta, Faculdade de Enfermagem, Centro de Ensino Superior de Valença. Rio de Janeiro, Brasil. E-mail: ana.munhen@faa.edu.br "Enfermeira. Doutora. Professora Titular, Faculdade de Enfermagem, Universidade do Estado do Rio de Janeiro. Brasil. E-mail: dcouerj@gmail.com

"'Enfermeira. Doutora, Programa de Pós-Graduação em Enfermagem da Universidade do Estado do Rio de Janeiro. Brasil. E-mail: virginiafigueiredo@yahoo.com.br IVEnfermeira. Doutoranda, Programa de Pós-graduação em Enfermagem da Universidade do Estado do Rio de Janeiro. Brasil. E-mail: yndiramachado@gmail.com ${ }^{\vee}$ Enfermeira. Mestre. Programa de Pós-graduação em Enfermagem da Universidade do Estado do Rio de Janeiro. Brasil. E-mail: renata_350@hotmail.com

v'Enfermeiro. Doutor. Professor Adjunto, Faculdade de Enfermagem, Centro de Ensino Superior de Valença. Rio de Janeiro, Brasil. E-mail: marcio.martins@faa.edu.br 
O risco biológico é destacado no ambiente hospitalar devido a permanente exposição dos trabalhadores de enfermagem aos agentes biológicos existentes no ambiente. Esse risco tornou-se mais evidente após o surgimento da Síndrome da Imunodeficiência Adquirida (AIDS) e do crescimento do número de pessoas infectadas pelos Vírus da Hepatite B e C4.

Nos serviços de saúde, grande parte dos acidentes que envolvem os trabalhadores se deve a não observância das normas de segurança, uma vez que o emprego de práticas seguras e o uso de equipamentos de proteção individual (EPIs) adequados reduzem significativamente o risco de acidente ocupacional ${ }^{5}$. A biossegurança compreende um conjunto de ações a fim de prevenir, controlar ou eliminar riscos inerentes às atividades que possam interferir ou comprometer a qualidade de vida, a saúde humana e o meio ambiente. As ações de biossegurança em saúde são primordiais para a promoção e manutenção do bem estar e proteção à vida e à saúde, assegurados nos princípios básicos do Sistema Único de Saúde (SUS) e nas políticas governamentais ${ }^{6}$.

No Brasil, a primeira legislação de biossegurança, Lei no 8794, foi criada em 1995. Em 2005, o Ministério do Trabalho instituiu a Norma Regulamentadora 32, que tem por finalidade estabelecer as diretrizes básicas para a implementação de medidas de proteção à segurança e à saúde dos trabalhadores dos serviços de saúde7,8. Em 2011, alguns artigos desta Norma sofreram alterações por meio da Portaria GM № 1.748/11, instituindo a obrigatoriedade da implantação de um Plano de Prevenção de Riscos de Acidentes com Materiais Pérfurocortantes ${ }^{9}$.

Dessa forma, este estudo objetiva analisar as representações sociais de biossegurança construídas pelos enfermeiros que atuam com pacientes soropositivos ao Vírus da Imunodeficiência Humana (HIV).

\section{RefERENCIAL TeÓRICO-MetOdológICo}

Estudo exploratório, descritivo, de abordagem qualitativa. Como referencial teórico metodológico utilizou-se a Teoria de Representações Sociais em sua abordagem processual ${ }^{10,11}$. Foram selecionadas oito instituições públicas de saúde, sendo quatro hospitais e quatro centros municipais de saúde, localizados no município do Rio de Janeiro, que prestam atendimentos a pessoas que vivem com o vírus do HIV e com a AIDS (PVHA).

Participaram 19 enfermeiras atuantes na atenção direta a PVHA. Cada participante recebeu uma nomenclatura fictícia, caracterizados como E1, E2 até E19. A coleta de dados ocorreu no ano de 2011, instrumentalizada por um roteiro temático de entrevista e um questionário de caracterização socioprofissional. Os dados socioprofissionais não serão apresentados neste estudo.

Foram respeitados os aspectos éticos de pesquisas, de acordo com a Resolução no 196/1996, vigente no período de coleta de dados. O projeto guarda-chuva ao qual este estudo está associado foi submetido ao Comitê de Ética em Pesquisa da Universidade do Estado do Rio de Janeiro (UERJ) e da Prefeitura do Rio de Janeiro, aprovados com os números de protocolo 048.3.2010 e 38A/2011, respectivamente.

As entrevistas foram submetidas à análise lexical, desenvolvida pela técnica hierárquica descendente do software ALCESTE 4.10. Foi construído um corpus que reúne as entrevistas transcritas num arquivo único, separadas pela linha estrelada contendo as variáveis referentes a cada uma delas. Após, o software procedeu a segmentação e tratamento do corpus. A finalidade de analisar o corpus por meio desta técnica quantitativa de tratamento de dados textuais é fornecer informações importantes sobre a natureza dos conteúdos da representação analisada ${ }^{12}$.

\section{RESULTADOS}

A Classificação Descendente Hierárquica (CDH) permite a visualização do corpus em conjuntos de Unidades de Contexto Elementar (UCE), caracterizadas por trechos de falas dos participantes. O conjunto de UCEs resultam em classes lexicais, que mostram os conteúdos de uma dada representação ou de mais de uma representação do objeto estudado. Ainda, como resultado de cada classe, apresenta-se a Classificação Ascendente Hierárquica (CAH), que se constitui pela determinação do valor de associação entre as formas reduzidas de uma determinada classe.

A análise do conjunto de entrevistas resultou em 6 classes identificadas na análise standard a partir da $\mathrm{CDH}$, sendo que destas, a classe 2 foi a que sintetizou os conteúdos relativos a biossegurança, sendo discutida neste trabalho a partir da $\mathrm{CAH}$.

A classe 2 - Medidas de precaução adotadas por enfermeiras que atuam no cuidado a pessoas que convivem com HIV/AIDS: o limiar entre a vida profissional $e$ pessoal - trata dos conteúdos relativos à biossegurança; assim, buscou-se identificar as ações de proteção desenvolvidas pelos trabalhadores de enfermagem, na vida pessoal e profissional, constituintes da representação que o grupo possui acerca da biossegurança, e que influenciam a prática profissional.

Esta classe abarca um total de 349 UCE, do total de 1.414 , representando $17 \%$ do corpus analisado. Apresenta associação estatística com: enfermeiras com idade entre 35 e 44 anos; contato físico e verbal com pessoas HIV positivo; frequência de contato diário com PVHA; tempo de atuação com PVHA de 11 a 15 anos.

As formas reduzidas associadas a esta classe, e que apresentaram maiores valores de quiquadrado (x2), foram: camisinha+; usa+; luva_de_proct; confit; relacionat; mascara; acidente_det; furt. Observa-se que as formas reduzidas apontam para conteúdos rela- 
cionados com medidas de precaução, na vida pessoal e profissional, bem como para a exposição ocupacional.

A delimitação dos conteúdos da classe 2 pode ser identificada de forma detalhada a partir da $\mathrm{CAH}$, proveniente das divisões binárias sucessivas efetuadas pelo software no material discursivo. O agrupamento das palavras que atribuem sentido à classe é especificado na Figura 1.

A partir do agrupamento das formas reduzidas e da análise das UCE da classe, foi possível identificar duas facetas relacionadas às medidas de biossegurança adotadas por enfermeiras em suas atividades cotidianas, que são descritas a seguir.

\section{O desenvolvimento da biossegurança em HIV/AIDS na vida pessoal}

As enfermeiras entrevistadas demonstram possuir conhecimento teórico relacionado às medidas de biossegurança e associam, em seus discursos, a autoproteção na vida pessoal com a importância do uso do preservativo, mesmo diante de uma relação considerada estável.

Quando eu comecei a ter noção do que era realmente a AIDS, comecei a pensar muito mais em proteção, em camisinha. Na minha época de juventude, falávamos que era 'chupar bala com papel'. E, sem dúvida mudou sim, mudou até mesmo em relação ao casamento. Porque não é por você estar casada que está imune. Isso também era uma coisa que eu acreditava e não acredito mais, não acredito mesmo. (E2)

Marcando a complexa relação estabelecida entre conhecimentos e práticas, alguns fatores foram descritos pelas enfermeiras como justificativa para a não adoção de preservativos, mesmo reconhecendo a sua necessidade. Entre eles, podem ser destacadas a confiança na fidelidade do parceiro, determinando uma sensação de invulnerabilidade à AIDS, e a dificuldade de negociação do uso do preservativo.

Acabamos correndo esse risco. Para mim não mudou porque eu não saio, não tenho relações fora do casamento e tento confiar muito no meu marido. (E6)

Eu falei: gente isso aí é muito difícil. Há pessoas que se você perguntar: você usa camisinha? Uso. Você vai dizer que não, que não acredita? Porque se as pessoas dizem que usam, é porque usam. Tem gente que confia. Eu confio no meu marido. (E9)

Na suspeita de relacionamentos extraconjugais, a necessidade de proteção é ressaltada e a dificuldade na negociação do uso do preservativo se faz presente.

De vez em quando eu fico pensando assim: será que ele [o parceiro] está direitinho? Eu sei de mim, mas nunca sabemos o que se passa na cabeça do outro, o que ele está fazendo fora do alcance da minha vista. Eu já tentei propor de ficar só com camisinha, mas para mim são mais complicadas essas coisas. (E3)

\section{Biossegurança em HIV/Aids e a execução do cuidado de enfermagem}

De forma geral, observa-se que os enfermeiros possuem conhecimentos teóricos relacionados à prevenção, precaução padrão, biossegurança e aos riscos ocupacionais a que estão expostos. No âmbito profissional, a autoproteção abarca questões relacionadas às precauções padrão e à exposição ocupacional, uma vez que reconhecem a importância da adoção de medidas de precaução para evitar a ocorrência de acidentes de trabalho durante a execução do cuidado.

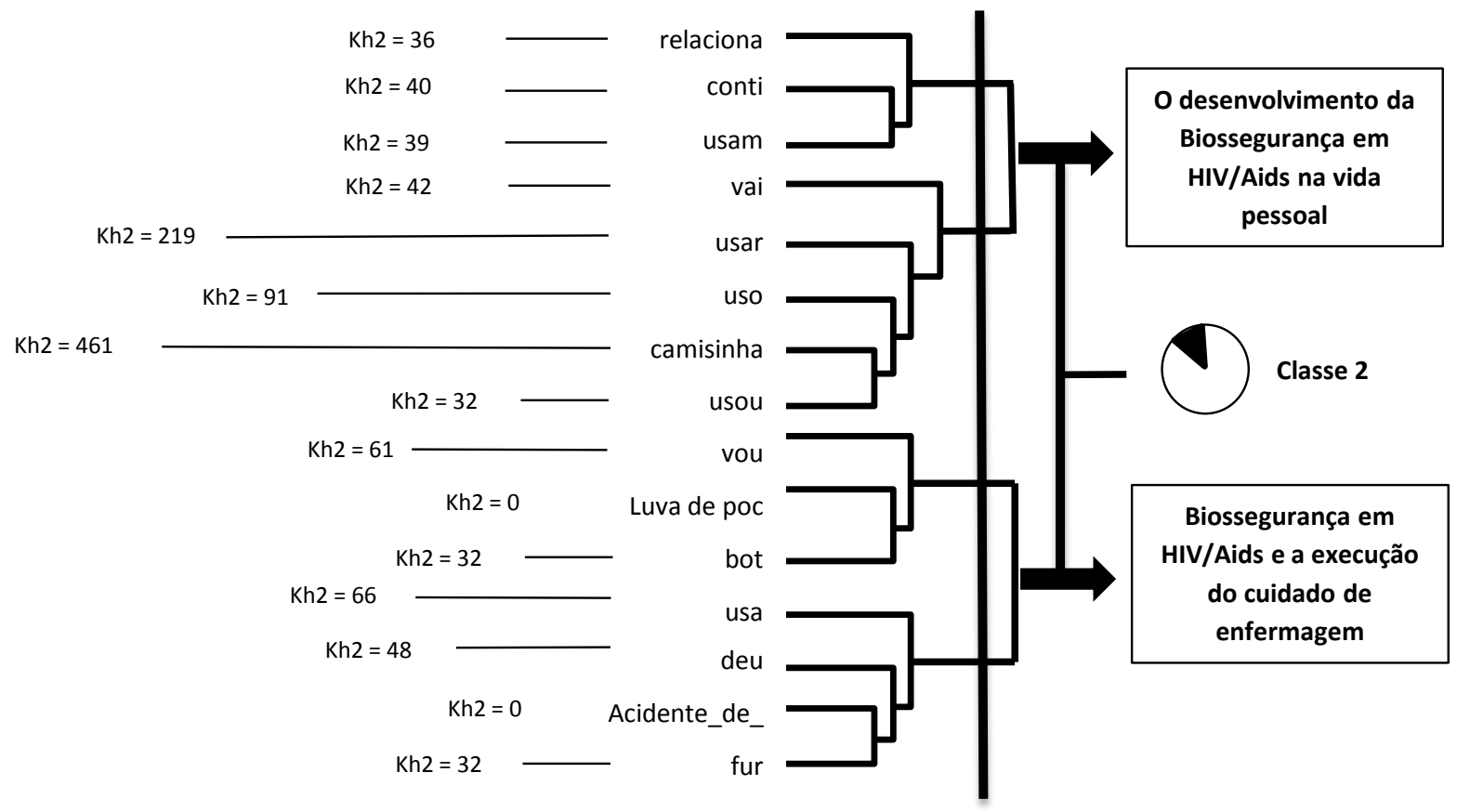

FIGURA 1: Classificação Ascendente Hierárquica da Classe 2. Rio de Janeiro, 2011. 
Porque aí é para usar eu uso. Que eu uso a luva de procedimento, eu uso a máscara, eu uso capote. Está calor, estou suando, o suor está pingando, mas eu estou lá. (E4)

Ele ia ali, ele se protegia em termos de equipamento de proteção individual. Quando você não sabe o que é, você usa tudo, você usa capote, máscara, um monte de coisas. Você não sabe que fluido orgânico contamina. (E3)

O cuidado de enfermagem é modulado pela presença conhecida do HIV, à medida que é referida maior cautela por parte das profissionais relacionada à ocorrência de acidentes com material biológico nessas situações. Sentimentos como medo, angústia e preconceito ao lidar com pacientes que vivem com HIV/AIDS estão presentes nos discursos.

Se eu sei que o paciente é um paciente HIV positivo ou sei que o paciente tem hepatite é claro que o meu cuidado é maior, até porque o paciente é uma fonte de contaminação, você vai com mais cuidado. (E3)

As enfermeiras reconhecem a necessidade e importância da utilização de EPI durante o cuidado de enfermagem, com destaque para a luva de procedimento, conforme evidenciado também em outro estudo ${ }^{13}$.

Nós sabemos que é comprovado cientificamente que a luva de procedimento é uma maneira [de proteção], eu uso sempre e falo para o pessoal novo. Nós temos, não falta, tem tudo. Agora, nem sempre as pessoas usam, eu mesmo não uso a máscara, estou sendo sincera [...]. (E9)

De forma pontual, foi possível identificar justificativas para o não uso de EPIs e para a ocorrência de acidentes. Entre elas sobressaem a proteção espiritual e algumas atividades que exigem ação imediata, como as situações de emergência.

Um acidente de trabalho pode acontecer com qualquer um, podendo usar a luva de procedimento, por que não usa? Aí ela falou assim: 'não é questão de ser acidente de trabalho, mesmo que eu me fure não tem problema, porque Nossa Senhora sempre está comigo' (E1).

Eu trabalhei muito, muito tempo mesmo lidando com contato direto com sangue de paciente. Porque dizem: porque não calça luva de procedimento? Às vezes a coisa é tão rápida, tão imediata, ou você faz ou o cara vai esvair em sangue ali e quando você viu, você já pegou $e$, as vezes, você não vê e encosta e tal... (E15)

As enfermeiras referem grande preocupação com os diversos tipos de acidentes aos quais estão expostas, destacando o risco biológico objetivado como acidente de trabalho com material biológico ou perfurocortante.

[falando sobre o receio de trabalhar no setor de infectologia] Daqui eu não sei quanto tempo, pode ser uma hipótese, é uma hipótese, e as pessoas têm medo disso e têm medo do acidente de trabalho com material biológico também, mas falamos que em qualquer lugar o cuidado de enfermagem é o mesmo. (E12)

Eu tive colegas que sofreram acidente de trabalho, furaram o dedo na hora de puncionar a veia, mas eu, graças a Deus, até hoje não me aconteceu isso. E também eu tenho o maior cuidado, e eu tenho que ter cuidado comigo e cuidado com os outros. (E8)

\section{DISCUSSÃO}

Sobre a temática contida no primeiro eixo, o desenvolvimento da biossegurança em HIV/AIDS na vida pessoal, emergiram falas sobre o conhecimento teórico das enfermeiras acerca das medidas de autoproteção. Observou-se que a prática profissional com pacientes HIV positivos traz implicações para a vida privada, pois os discursos dos participantes são caracterizados pela assunção de práticas sexuais mais seguras nas relações íntimas, uso do preservativo e a necessidade do diálogo no seio familiar.

Os relatos de não utilização do preservativo associado à crença na fidelidade do parceiro remetem a um processo representacional onde o casamento assume o lugar das medidas de proteção contra o HIV/AIDS. Nessa perspectiva, algumas mulheres acreditam na invulnerabilidade à infecção como extensão da crença na fidelidade do parceiro.

A confiabilidade e o tempo de relacionamento são considerados fatores importantes na decisão pelo não uso do preservativo ${ }^{14}$. Dessa forma, a confiança no parceiro associa-se a práticas de sexo não seguro, o que potencializa o risco de infecção pelo HIV, na existência de relacionamentos extraconjugais desconhecidos ${ }^{14,15}$

Em 1987 foi publicado o Guia para Prevenção da Transmissão do HIV e Vírus da Hepatite B nos Profissionais de Saúde, pelo Center for Diseases Control and Prevention (CDC). As medidas de Precaução Padrão (PP) são preconizadas pelo CDC que orienta a sua utilização para todos os pacientes, independente da sua patologia, em todos os procedimentos que envolvam fluidos corpóreos que possuam sangue ${ }^{16}$.

Acerca do segundo eixo, biossegurança em HIV/ AIDS e a execução do cuidado de enfermagem, observase que o processo de trabalho de enfermagem está associado a diversos riscos e vulnerabilidades, dentre eles encontram-se os riscos biológicos e de acidentes. Há o reconhecimento da importância da precaução padrão durante a realização do cuidado de enfermagem. Vale enfatizar que esta precaução deve ser adotada para todos os pacientes, independente de sua doença.

Entretanto, diversas profissionais referiram a realização de adequações do cuidado de enfermagem prestado ao paciente sabidamente HIV positivo, em relação aos demais. Estas particularidades são caracterizadas pela utilização excessiva de cuidados quando em exposição ocupacional ao $\mathrm{HIV}^{17}$, contrariando o preconizado pelo $\mathrm{CDC}^{16}$.

Importantes alterações ocorreram nas práticas profissionais das enfermeiras devido aos processos de explicação que se estabeleceram frente a AIDS. Entre as mudanças pode-se ressaltar a objetivação de práticas de distanciamento, verificadas tanto no uso de técnicas de autoproteção profissional desnecessárias, quanto no distanciamento observado nas relações interpessoais com os pacientes ${ }^{18}$. 
Para essas enfermeiras, os EPIs são necessários e têm sua importância reconhecida durante o cuidado de enfermagem. Destaca-se a luva de procedimento, em detrimento de outros, como o uso de máscara e de óculos de proteção, na dependência da situação de exposição. No estudo observou-se que a luva foi citada como o principal EPI, e por vezes o único, sendo consequentemente o mais utilizado pelas profissionais na redução da exposição ocupacional no contato com sangue e fluidos corpóreos.

Vale realçar que a aplicação das medidas de biossegurança está relacionada com o controle dos riscos aos quais os profissionais de saúde se encontram expostos. Entre estas medidas sobressaem o uso de EPI, a educação continuada, higienização correta das mãos, e, sobretudo, a escolha das medidas adequadas conforme a situação de exposição enfrentada pelo profissional.

A higienização das mãos, segundo a Organização Mundial de Saúde (OMS), é uma prática simples e muito importante como medida de biossegurança e na prevenção e controle da infecção hospitalar. No entanto, ainda existe uma enorme dificuldade de colocá-la em prática ${ }^{19}$. Neste estudo, nenhuma enfermeira se referiu à lavagem das mãos como uma medida de biossegurança; isso reforça que o conhecimento e as práticas dos profissionais em relação à biossegurança nem sempre condizem com os cuidados recomendados pelas normas técnicas.

A biossegurança constitui-se como uma recente área do saber, o que representa um desafio para os profissionais de saúde. Observa-se uma lacuna existente entre o campo teórico e a prática diária, uma vez que em diversos contextos as normas de biossegurança não são aderidas adequadamente ${ }^{20}$.

As situações que justificam o não uso de EPIs no cotidiano hospitalar tornam os profissionais mais vulneráveis às condições do ambiente de trabalho, aos acidentes com materiais biológicos e, ainda, à contaminação por uma doença transmissível. A vulnerabilidade referida é subestimada pelos trabalhadores de enfermagem, levando a um sentimento de invulnerabilidade potencializado pela crença na proteção espiritual.

Estudos apontam que os acidentes de trabalho ocorrem por diversos motivos, tais como: o recapeamento de agulha, a não utilização de EPIs, a falta de atenção do profissional, o ritmo de trabalho intenso e as condições de trabalho. A inadequação dos EPIs também foi apontada como um dos principais problemas que predispõem ao acidente. Verifica-se que a maioria dos trabalhadores resiste ao correto uso do EPI, aumentando a exposição a acidentes por material biológico ${ }^{3,13,21}$.

Ao longo do tempo, a adoção das medidas de biossegurança nas atividades profissionais tem se apresentado como um desafio para a enfermagem. Observa-se certo conhecimento e aceitação das normas de biossegurança no discurso das profissionais, contudo, elas não são incorporadas à prática diária com a mesma intensidade, revelando a complexa trama e a não horizontalidade das relações entre conhecimentos e práticas ${ }^{22}$.

Os sentimentos como medo e preocupação permeiam o cotidiano dos profissionais de saúde que temem sofrer um acidente no trabalho, mesmo entre aqueles que nunca se acidentaram. Esses aspectos revelam a intensidade das repercussões psicológicas e sociais do acidente no ambiente de trabalho. Fato este que reforça a necessidade do reconhecimento e da inclusão das dimensões psicossociais e educativas nos programas de prevenção de acidentes e assistência aos trabalhadores nas instituições de saúde ${ }^{13}$.

Através do questionário de caracterização aplicado, percebeu-se uma fragilidade da capacitação profissional, visto que a maioria das enfermeiras inclusas neste estudo nunca participou de cursos específicos voltados para o tema HIV/AIDS. Considera-se incontestável a importância da discussão sobre biossegurança na formação profissional de estudantes da área da saúde e a atualização constante dos profissionais de enfermagem, nessa e demais áreas, uma vez que a qualificação da força de trabalho representa uma das principais necessidades para a incorporação de medidas de biossegurança à prática assistencial ${ }^{23}$.

\section{CONCLUSÃO}

A discussão sobre biossegurança apresenta-se hoje mais presente nas instituições de saúde do país, porém esta discussão ainda não foi incorporada de forma plena pelos trabalhadores que atuam na assistência direta ao paciente. Os participantes do estudo conhecem as medidas de biossegurança, referem-se a elas, no entanto, ainda não as transformaram em práticas profissionais contínuas e conscientes.

A representação social da biossegurança se constitui num conjunto de saberes, atitudes, condutas e imagens decorrentes da vivência cotidiana dos profissionais nos locais de trabalho, assim, percebe-se que a própria transmissibilidade da AIDS e de outras doenças como a Hepatite B e C, são retraduzidas nessa representação. Se, por um lado, essa transmissibilidade é reconhecida e nomeada em nível cognitivo, por outro ela não se expressa da mesma forma em nível das práticas profissionais.

Acerca do pensamento teórico, a transmissibilidade se expressa em risco à integridade física do enfermeiro; acerca do pensamento prático, ela passa a se constituir em possibilidade vaga de comprometimento dessa integridade, resultando em comportamentos alternativos de proteção.

Assim, pode-se inferir a hipótese de existência de uma representação não autônoma da biossegurança, ancorada na representação social da AIDS. Observa-se que a contradição entre medo do contágio e sensação de invulnerabilidade ao HIV condicionam as práticas, 
as atitudes e as condutas de proteção adotadas pelos profissionais estudados.

Apresenta-se como limitação deste estudo, o número de participantes, o que permite sugerir uma ampliação da amostra, bem como a adoção de uma única técnica de análise, uma vez que no campo das representações sociais a triangulação de dados permitiria uma compreensão mais ampliada do objeto de estudo em questão.

Por fim, acredita-se que programas de educação continuada/permanente sobre saúde do trabalhador e núcleos de vigilância hospitalar possam ser grandes aliados na implantação das normas de biossegurança nos serviços de saúde, a partir de uma participação efetiva e responsável dos profissionais, de forma a melhorar as condições de trabalho, tornando-o seguro e distante de riscos.

\section{REFERÊNCIAS}

1. Marziale MHP, Rocha FLR, Robazzi MLCC, Cenzi CM, Santos HEC, Trovó MEM. Organizational influence on the occurrence of work accidents involving exposure to biological material. Rev. latinoam. enferm. [Online]. 2013; [cited 2018 Apr 20]; 21(Spec): [08 telas]. Available from: http://www.scielo.br/pdf/rlae/v21nspe/pt_25.pdf 2. Carvalho DC, Rocha JC, Gimenes MCA, Santos EC, Valim MD. Acidentes de trabalho com materiais biológicos na equipe de enfermagem de um hospital do Centro-Oeste brasileiro. Esc. Anna Nery Rev. Enferm. [Online]. 2018; [citado em 16 jan 2018]; 22(1): 1-8. Disponível em: http://www.scielo.br/pdf/ean/v22n1/ pt_1414-8145-ean-2177-9465-EAN-2017-0140.pdf

3. Oliveira AC, Paiva MHRS. Análise dos acidentes ocupacionais com material biológico entre profissionais em serviços de atendimento pré-hospitalar. Rev. latino-am. Enferm. [Online]. 2013 [citado em 18 abr 2018]; 21:309-15. Disponível em: http://www. scielo.br/pdf/rlae/v21n1/pt_v21n1a04.pdf

4. Magagnini MAM, Rocha SA, Ayres JA. O significado do acidente de trabalho com material biológico para os profissionais de enfermagem. Rev. gaúch. enferm. 2011 [citado em 20 abr 2018]; 32(2):302-8. Disponível em: http://www.scielo.br/pdf/rgenf/ v32n2/a13v32n2.pdf

5. Valle ARMC, Feitosa MBF, Araújo VMD, Moura MEB, Santos AMR, Monteiro CFS. Representações sociais da biossegurança por profissionais de enfermagem de um serviço de emergência. Esc. Anna Nery Rev. Enferm. 2008; 12(2): 304-9.

6. Ministério da Saúde (Br). Biossegurança em saúde: prioridades e estratégias de ação. [online]. Brasília (DF); 2010. [citado 20 abr 2018] Disponível em: http://bvsms.saude.gov.br/bvs/publicacoes/ biosseguranca_saude_prioridades_estrategicas_acao_p1.pdf

7. Ministério do Trabalho e Emprego ( $\mathrm{Br}$ ). Riscos biológicos: guia técnico. Os riscos biológicos no âmbito da Norma Regulamentadora no 32. Brasília: MTE; 2008.

8. Manuais de Legislação. Segurança e medicina do trabalho. São Paulo: Atlas; 2008.

9. Ministério do Trabalho e Emprego (Br). Portaria GM n. 1.748 de 31 de agosto de 2011. Altera a Norma Regulamentadora (NR) no 32. Diário Oficial da União. 31 ago 2011, Art. 1ㅇ. [citado 4 jul 2012]. Disponível em: http://seesspa.com.br/site/wp-content/ uploads/2011/12/PORTARIA-N.\%C2\%BA-1.748-DE-30-DE-AGOSTO-DE-2011.pdf

10. Jodelet D. Representação social: um domínio em expansão In: Jodelet $D$, organizadora. As representações sociais. Rio de Janeiro: Ed. UERJ; 2001. p. 17-44.

11. Sá CP. A construção do objeto de pesquisa em representações sociais. Rio de Janeiro: Ed. UERJ; 1998.

12. Oliveira DC, Gomes AMT, Marques SC. Análise estatística de dados textuais na pesquisa das representações sociais: alguns princípios e uma aplicação no campo da saúde. In: Menin MSS, Shimizu AM., organizadores. Experiência e representação social: questões teóricas e metodológicas. São Paulo: Casa do Psicólogo; 2005. p.157-200

13. Sousa AFL, Queiroz AAFLN, Oliveira LB, Moura MEL, Batista OMA, Andrade D. Representações sociais da enfermagem sobre biossegurança: saúde ocupacional e o cuidar prevencionista. Rev. bras. enferm. [Online]. 2016 [citado em 18 fev 2018]; 69(5):86471. Disponível em: http://www.scielo.br/scielo.php?script=sci_ arttext\&pid=S0034-71672016000500864\&Ing=en.

14. Felix G, Ceolim MF. The profile of women with HIV/AIDS and their adherence to the antiretroviral therapy. Esc. Enferm. USP. 2012 [cited 2018 Apr 20]; 46(4):884-91. Available from: http:// www.scielo.br/pdf/reeusp/v46n4/15.pdf

15. Carvalho CM.L, Martins LFA, Galvão MTG. Sentimentos de muIheres portadoras de HIV/AIDS diante da percepção da infecção. Revista Nursing. 2006; 100(8):1010-5.

16. Centers for Disease Control and Prevention (CDC). Guide to infection prevent for out patient settings: minimum expectations for safe care. [Internet]. 2015 [cited 2018 Apr 08]; Available from: https://www.cdc.gov/hai/settings/outpatient/outpatient-care-guidelines.html

17. Formozo GA, Oliveira DC. Autoproteção profissional e cuidado de enfermagem ao paciente sororpositivo ao HIV: duas facetas de uma representação. Acta paul. enferm. 2009; 22(4):392-8

18. Oliveira DC, Erdman AL, Giami A, Vargens OMC, Oliveira SA, Gomes AMT, et al. Representações e memória profissional da AIDS de enfermeiras no Brasil: estudo bicêntrico Rio de Janeiro/ Florianópolis. Psicologia teoria e prática. 2007; 9(1): 114-8.

19. Ministério da Saúde (Br). Higienização das mãos em serviços de saúde. Brasília (DF): Anvisa; 2007.

20. Oliveira JS, Macedo MP, Morais RLGL, Tonan MS, Yarid SD. Biossegurança sob a ótica dos graduandos de enfermagem. Rev. enferm. UERJ. 2017; [citado em 22 fev 2018]; 25: [e14074]: 1-5. Disponível em: https://www.e-publicacoes.uerj.br/index.php/ enfermagemuerj/article/view/14074

21. Villarinho MV, Padilha MICS. Risco ao acidente de trabalho no cuidado às pessoas internadas por HIV/AIDS, Florianópolis-SC-Brasil (1986-2006). Rev. enferm. UFSM. 2014;4(4):689-99.

22. Oliveira AC, Paiva MHRS. Prevalência e características dos acidentes com material biológico envolvendo profissionais do atendimento pré-hospitalar móvel. Cienc. cuid. saúde [Online]. 2013; [citado em 20 abr 2018]; 12(2):323-30. Disponível em: http://ojs. uem.br/ojs/infex.php/CiencCuidSaude/article/viewFile?19371/pdf 23. Marziale MHP, Santos HEC, Trovó MEM. Consequências individuais e ocupacionais da exposição a material biológico entre trabalhadores de enfermagem. Rev. enferm. UERJ. [Online]. 2015 [citado em 12 abr 2018]; 23(4):449-54. Disponível em: http:// www.facenf.uerj.br/v23n4/v23n4a03.pdf 